\section{Interaction of vitamin $E$ isoforms on asthma and allergic airway disease}

\footnotetext{
Abstract Prospective epidemiological studies, observational cross-sectional studies and some randomised prevention trials have

demonstrated inconsistent findings of the impact of vitamin $\mathrm{E}$ on asthma risk. The goals of this study were to explore whether this differing association of vitamin $E$ on asthma risk is due to an interaction of vitamin $\mathrm{E}$ isoforms. To address this question, in a population-based asthma incidence study we assessed the interaction between the plasma concentrations of vitamin $\mathrm{E}$ isoforms $\alpha$-tocopherol and $\gamma$-tocopherol on asthma risk. Second, to understand the mechanisms of any interaction of these isoforms, we conducted experimental supplementation of $\alpha$-tocopherol and $\gamma$-tocopherol isoforms in mice on the outcome of allergic airway inflammation. We found that in the highest $\gamma$-tocopherol tertile, low levels of $\alpha$-tocopherol were associated with increased asthma risk, while highest tertile $\alpha$-tocopherol levels trended to be protective. Similarly, in a mouse model of asthma, diet supplementation with $\alpha$-tocopherol decreased lung inflammation in response to house dust mite (HDM) challenge. In contrast, diet supplementation with $\gamma$-tocopherol increased lung inflammation in response to HDM. These human and animal studies provide evidence for the competing
}

effects of the vitamin $E$ isoforms, in physiological concentrations, on asthma and allergic airway disease.

\section{INTRODUCTION}

Prospective epidemiological studies, observational cross-sectional studies and some randomised prevention trials have demonstrated the impact of vitamin $\mathrm{E}$ on asthma risk. However, the directionality of the findings has not been consistent. Studies of vitamin $\mathrm{E}$ have sometimes included total vitamin $\mathrm{E}$, without evaluating isoform concentrations, and have been conducted both over time and in different countries where the food isoform content of $\alpha$-tocopherol and $\gamma$-tocopherol vary widely. In a recent prospective incidence study of the role of dietary antioxidants on asthma inception, we found a protective association for $\alpha$-tocopherol, but not with $\gamma$-tocopherol. To address these divergent results and investigate the mechanisms through which vitamin E may promote or protect against asthma development, we report on the interaction of $\alpha$-tocopherol and $\gamma$-tocopherol on asthma risk in a human cohort study, and the effect of experimental supplementation of individual isoforms on allergic airway inflammation in an animal model.

\section{METHODS}

To explore whether this differing association of vitamin $\mathrm{E}$ isoforms on asthma risk is due to an interaction of these isoforms and to follow-up these findings to identify differential mechanisms and in vivo interaction, we conducted two studies. First, in the Shanghai Women's Asthma and Allergy Study, the only known study designed and powered to investigate the association of antioxidant defences on adult incident asthma, we assessed the interaction between $\alpha$-tocopherol and $\gamma$-tocopherol in this population-based asthma incidence cohort on asthma risk. ${ }^{12}$ Second, to understand the mechanisms of any interaction of these isoforms, we conducted experimental supplementation of $\alpha$-tocopherol and $\gamma$-tocopherol isoforms in mice on the outcome of allergic airway inflammation. The detailed methods of these studies are included in the online supplementary material.

\section{RESULTS}

The interaction of $\alpha$-tocopherol and $\gamma$-tocopherol was assessed as continuous variables using multivariable conditional logistic regression analysis, while adjusting

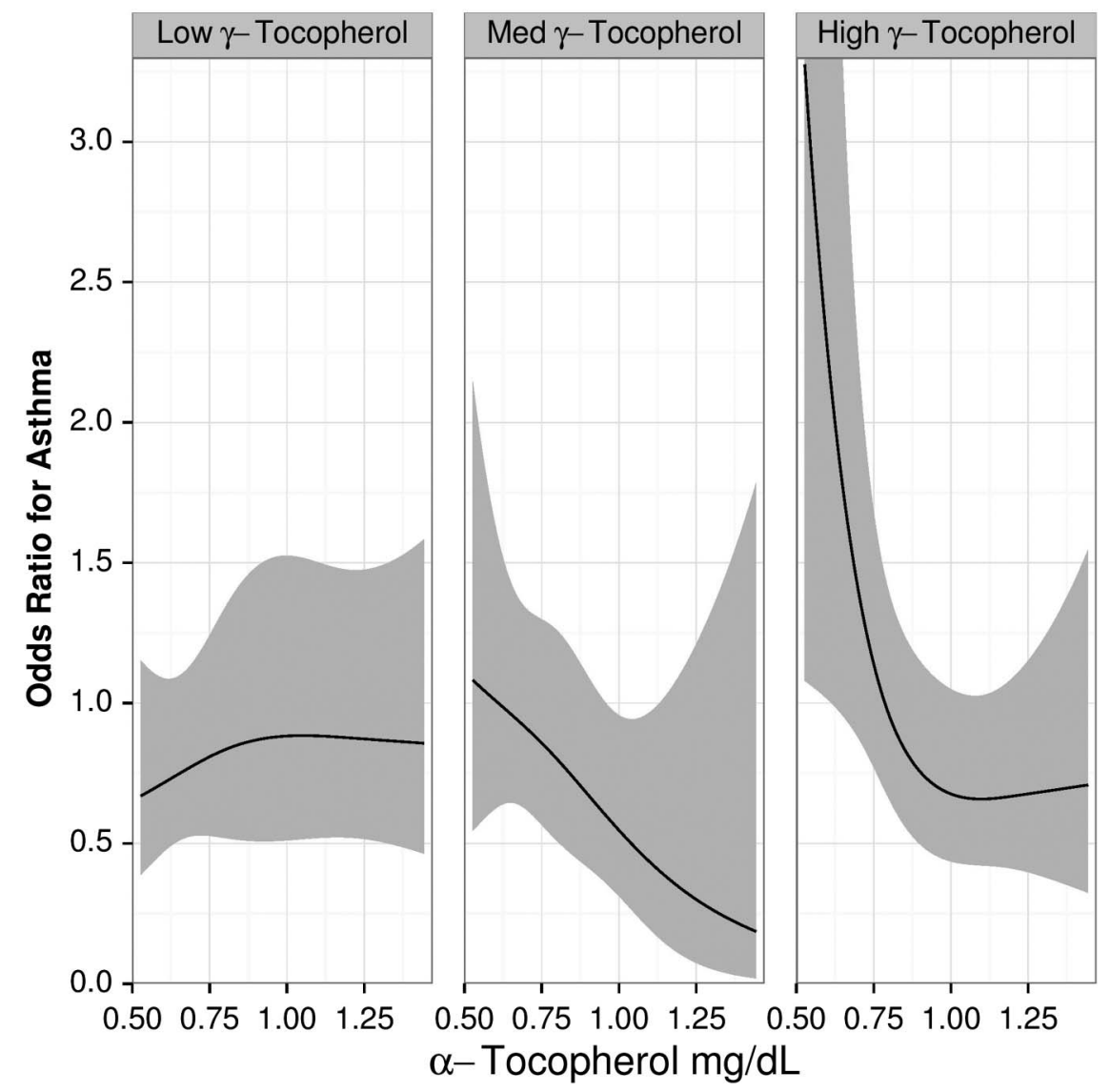

Figure 1 Interaction of plasma levels of $\alpha$-tocopherol and $\gamma$-tocopherol on asthma risk. 


\section{$\alpha$-Tocopherol supplementation}

\section{A Inflammatory Cells}

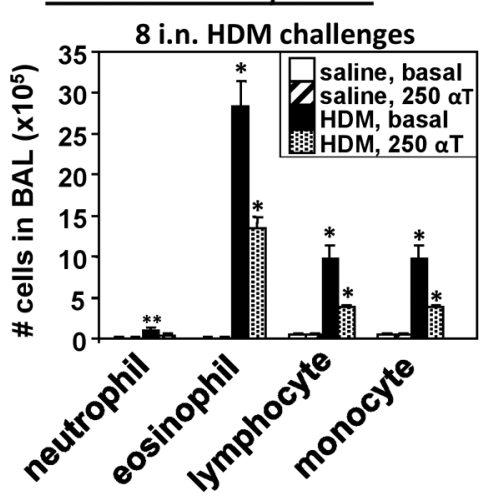

B Cytokines

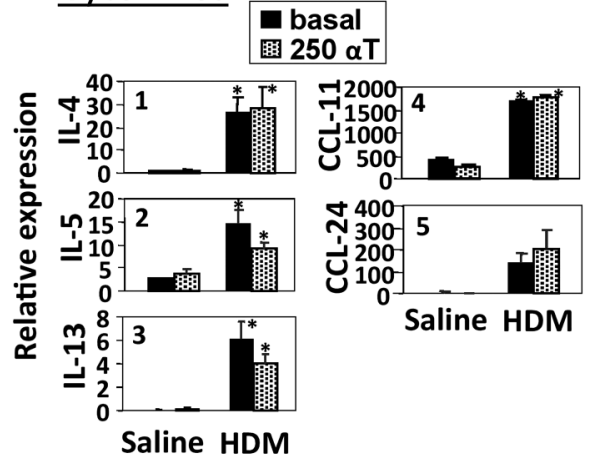

inflammation. In the human cohort study, we observed effect modification of the protective relationship of $\alpha$-tocopherol on asthma risk at varying levels of $\gamma$-tocopherol. In the animal model, we demonstrated a differential response of supplementation of $\alpha$-tocopherol and $\gamma$-tocopherol on lung inflammation in response to HDM challenge, suggesting that these isoforms act in opposing directions on allergic airway responses.

Previous studies of vitamin E on asthma risk have been limited by assessing aggregate levels of isoforms or merely dietary intake across different areas of the world where the $\alpha$-tocopherol and $\gamma$-tocopherol content of diets is dramatically different. ${ }^{7}$ In addition, in supplementation trials, supraphysiological supplementation has been used, and in $\alpha$-tocopherol supplementation trials, the carrier is often high in $\gamma$-tocopherol, making it difficult to assess the independent effect of the isoforms. Clinically, $\alpha$-tocopherol has also been associated with higher lung function and $\gamma$-tocopherol associated with lower lung function. $^{8}$

This report is the first rodent study demonstrating that diet supplementation with $\alpha$-tocopherol inhibits and $\gamma$-tocopherol increases eosinophilic allergic inflammation to the clinically relevant antigen HDM. In previous studies with chicken egg ovalbu$\min (\mathrm{OVA})^{4}{ }^{5}$ and the current study with responses to HDM, daily administration of tocopherols did not significantly regulate cytokines and chemokines in the lung tissue. However, $\alpha$-tocopherol has been reported to inhibit, and $\gamma$-tocopherol increase, eosinophil migration across endothelium and into tissues during allergic responses. ${ }^{468}$ In mechanistic studies, we have demonstrated that $\alpha$-tocopherol is an antagonist of protein kinase $\mathrm{C}$ alpha (PKC $\alpha$ ) and that $\gamma$-tocopherol is an agonist of PKC $\alpha .^{6}$ PKC $\alpha$ is activated by vascular cell adhesion molecule-1 (VCAM-1) on endothelial cells during VCAM-1-dependent recruitment of eosinophils. ${ }^{9} 10$ Taken together, this suggests that tocopherol isoforms have opposing functions on the signals for eosinophil recruitment. However, there is no perfect clarity on the impact of these isoforms on lung inflammation in animal studies. In a study with intermittent oral gavage $\alpha$-tocopherol on HDM challenge, $\alpha$-tocopherol did not alter HDM-induced inflammation; importantly, however, the $\alpha$-tocopherol was administered after the HDM challenge. ${ }^{11}$ In other studies with daily subcutaneous tocopherol administration, which raises tissue tocopherols 2-3-fold, $\alpha$-tocopherol reduced eosinophilic inflammation while tertiles, asthma risk varied with increasing $\alpha$-tocopherol. In the medium and highest tertile $\gamma$-tocopherol, asthma risk fell
This set of human and animal studies helps elucidate the opposing roles of vitamin E isoforms on asthma and allergic airway 
$\gamma$-tocopherol increased eosinophils in OVA-induced responses that were primarily eosinophilic. ${ }^{45}$ In contrast in another study, $\gamma$-tocopherol supplementation reduced antigen induction of rat lung inflammation in which there was 2.5 -fold more neutrophils than eosinophils. ${ }^{12}$ It is known that in the presence of lipopolysaccharide (LPS) in OVA inhalations, the lung lavage becomes predominantly neutrophilic (2-3 times more neutrophils than eosinophils), while ${ }^{13}$ it is also reported that nebulised $\gamma$-tocopherol reduces neutrophilia in burn and smoke inhalation injury in sheep. ${ }^{14}$ In acute models of inflammation with large numbers of neutrophils, such as with LPS or ozone, there is generation of reactive nitric oxide species, known to be ${ }^{15} \quad 16$ scavenged by $\gamma$-tocopherol but not $\alpha$-tocopherol. ${ }^{17-19}$ Therefore, $\gamma$-tocopherol, which scavenges nitric oxide, may be of benefit for acute neutrophilic inflammation, whereas it may increase eosinophilia during allergic responses to OVA and HDM.

There are limitations of these studies that should be considered. The human study was not designed specifically to assess the interaction between the vitamin $\mathrm{E}$ isoforms. The animal studies may not simulate what occurs in humans nor account for the human host differences in vitamin E metabolism, and the animal studies were done with either one of the two isoforms to determine their independent effects as a follow-up to the interaction noted in the human study. Lastly, while animal models allow for testing in controlled settings where everything else can be held constant, it is well recognised that murine allergen challenge models do not replicate what occurs in humans. However, despite these limitations, the combination of a human asthma incidence study and a suggested mechanism in an animal model supports the idea of opposing effects of these vitamin $\mathrm{E}$ isoforms, which may also help to explain the discrepant findings among studies.

These human and animal studies provide evidence for potential competition of the vitamin $\mathrm{E}$ isoforms, in physiological concentrations, on asthma and allergic airway disease. Controlled clinical studies are needed to further elucidate the effects of modifying diet to decrease $\gamma$-tocopherol intake, enhance $\alpha$-tocopherol intake or supplementation of $\alpha$-tocopherol at physiological concentrations on the prevention of asthma and allergic airway disease.
Joan Cook-Mills, ${ }^{1}$ Tebeb Gebretsadik, ${ }^{2}$ Hiam Abdala-Valencia, ${ }^{1}$ Jeremy Green, ${ }^{1}$ Emma K Larkin, ${ }^{2}$ William D Dupont ${ }^{2}$ Xiao Ou Shu, ${ }^{2}$ Myron Gross, ${ }^{3}$ Chunxue Bai, ${ }^{4}$ Yu-Tang Gao, ${ }^{5}$ Terryl J Hartman, ${ }^{6}$ Christian Rosas-Salazar, ${ }^{2}$ Tina Hartert ${ }^{2}$

${ }^{1}$ Northwestern University, Chicago, Illinois, USA ${ }^{2}$ Vanderbilt University Medical Center, Nashville, Tennessee, USA

${ }^{3}$ Department of Laboratory Medicine and Pathology, School of Public Health, University of Minnesota, Minneapolis, Minnesota, USA

${ }^{4}$ Zhongshan Hospital, Fudan University, Shanghai, People's Republic of China

${ }^{5}$ Department of Epidemiology, Shanghai Cancer Institute, Shanghai, People's Republic of China ${ }^{6}$ Department of Epidemiology, Rollins School of Public Health, Emory University, Atlanta, Georgia, USA

Correspondence to Dr Joan Cook-Mills, Northwestern University Feinberg School of Medicine, 240 E. Huron, McGaw M304, Chicago, IL 60611, USA; j-cook-mills@northwestern.edu

Contributors JC-M was involved in study design, animal experiments, data analysis and writing the manuscript. TG and WDD were involved in study design, data analysis and editing the manuscript. HA-V and JG were involved in the animal experiments and data analysis. EKL, CR-S and TJH were involved in the human study, data analysis and editing the manuscript. XOS was involved in study design and study funding. $\mathrm{TH}$ was involved in study design, human study data acquisition, data analysis, writing the manuscript and study funding.

Funding This study was funded by National Institute of Allergy and Infectious Diseases R01 Al 50884 (TH), K24 Al $77930(\mathrm{TH})$, National Institute of Complementary and Alternative Medicine R01 AT004837 (JC-M) and National Institute of Heart, Lung, and Blood R01HL111624 (JC-M). The project described was also supported by the U.S. National Cancer Institute R37 (Dr Wei Zheng) and NO2-CP11010-66 (XOS).

Competing interests None declared.

Ethics approval Institutional Review Board.

Provenance and peer review Not commissioned; externally peer reviewed.

- Additional material is published online only. To view please visit the journal online (http://dx.doi.org/10. 1136/thoraxjn-2016-208494).

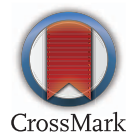

To cite Cook-Mills J, Gebretsadik T, AbdalaValencia H, et al. Thorax 2016;71:954-956.

Received 16 February 2016

Revised 5 May 2016

Accepted 8 May 2016

Published Online First 02 June 2016

Thorax 2016:71:954-956.

doi:10.1136/thoraxjnl-2016-208494

\section{REFERENCES}

1 Hartert TV, Deng X, Hartman TJ, et al. The Shanghai Women's Asthma and Allergy Study: objectives, design, and recruitment results. Am J Epidemiol 2008;167:1387-96.
2 Larkin EK, Gao YT, Gebretsadik T, et al. New risk factors for adult-onset incident asthma. A nested case-control study of host antioxidant defense. Am J Respir Crit Care Med 2015;191:45-53.

3 Abdala-Valencia H, Berdnikovs S, Cook-Mills JM. Vitamin $\mathrm{E}$ isoforms differentially regulate intercellular adhesion molecule- 1 activation of PKC $\alpha$ in human microvascular endothelial cells. PLOS ONE 2012;7: e41054.

4 Berdnikovs S, Abdala-Valencia H, McCary C, et al. Isoforms of vitamin $E$ have opposing immunoregulatory functions during inflammation by regulating leukocyte recruitment. J Immunol 2009;182:4395-405.

5 McCary CA, Abdala-Valencia H, Berdnikovs S, et al. Supplemental and highly elevated tocopherol doses differentially regulate allergic inflammation: reversibility of alpha-tocopherol and gamma-tocopherol's effects. J Immunol 2011;186:3674-85.

6 McCary CA, Yoon Y, Panagabko C, et al. Vitamin E isoforms directly bind PKC $\alpha$ and differentially regulate activation of PKC $\alpha$. Biochem J 2012:441:189-98.

7 Cook-Mills JM, Avila PC. Vitamin E and D regulation of allergic asthma immunopathogenesis. Int Immunopharmacol 2014;23:364-72.

8 Marchese ME, Kumar R, Colangelo LA, et al. The vitamin $E$ isoforms alpha-tocopherol and gamma-tocopherol have opposite associations with spirometric parameters: the CARDIA study. Respir Res 2014;15:31.

9 Abdala-Valencia H, Cook-Mills JM. VCAM-1 signals activate endothelial cell protein kinase Calpha via oxidation. J Immunol 2006;177:6379-87.

10 Cook-Mills JM. Isoforms of Vitamin E Differentially Regulate PKC $\alpha$ and Inflammation: A Review. J Clin Cell Immunol 2013;4.

11 Peh HY, Ho WE, Cheng C, et al. Vitamin E Isoform gamma-Tocotrienol Downregulates House Dust Mite-Induced Asthma. J Immunol 2015;195:437-44.

12 Wagner JG, Jiang Q, Harkema JR, et al. Gamma-tocopherol prevents airway eosinophilia and mucous cell hyperplasia in experimentally induced allergic rhinitis and asthma. Clin Exp Allergy 2008:38:501-11.

13 Tsuchiya K, Siddiqui S, Risse PA, et al. The presence of LPS in OVA inhalations affects airway inflammation and AHR but not remodeling in a rodent model of asthma. Am J Physiol Lung Cell Mol Physiol 2012;303:L54-63.

14 Hamahata A, Enkhbaatar P, Kraft ER, et al. gamma-Tocopherol nebulization by a lipid aerosolization device improves pulmonary function in sheep with burn and smoke inhalation injury. Free Radic Biol Med 2008;45:425-33.

15 Fakhrzadeh L, Laskin JD, Laskin DL. Ozone-induced production of nitric oxide and TNF-alpha and tissue injury are dependent on NF-kappaB p50. Am J Physiol Lung Cell Mol Physiol 2004;287:L279-85.

16 He $Y$, Franchi $L$, Núñez $G$. The protein kinase PKR is critical for LPS-induced iNOS production but dispensable for inflammasome activation in macrophages. Eur J Immunol 2013;43:1147-52.

17 Christen S, Woodall AA, Shigenaga MK, et al. gamma-tocopherol traps mutagenic electrophiles such as $\mathrm{NO}(X)$ and complements alpha-tocopherol: physiological implications. Proc Natl Acad Sci USA 1997;94:3217-22.

18 Patel A, Liebner $F$, Netscher $T$, et al. Vitamin E chemistry. Nitration of non-alpha-tocopherols: products and mechanistic considerations. J Org Chem 2007;72:6504-12.

19 Wolf G. gamma-Tocopherol: an efficient protector of lipids against nitric oxide-initiated peroxidative damage. Nutr Rev 1997;55:376-8. 